\title{
recillunds
}

Revista Científica Mundo de la Investigación y el Conocimiento

Tito Max Banegas Peña ${ }^{\text {a }}$, Jorge Edison Becerra Molina ${ }^{\text {b; }}$ Edgar Geovanny Zamora

$$
\text { Zamora }^{c} \text {; Héctor Espinoza Pillaga }{ }^{\mathrm{d}}
$$

Los modelos de equilibrio general dinámico estocásticos fundamentación microeconómica

Stochastic dynamic general equilibrium models microeconomic foundation

Revista Científica Mundo de la Investigación y el Conocimiento. Vol. 3 núm.3, septiembre, ISSN: 2588-073X, 2019, pp. 735-763

DOI: $10.26820 /$ recimundo/3.(3).septiembre.2019.735-763

URL: http://recimundo.com/index.php/es/article/view/537

Código UNESCO: 5302 Econometría

Tipo de Investigación: Artículo de Revisión

Editorial Saberes del Conocimiento

Recibido: 15/05/2019

Aceptado: 23/06/2019

Publicado: 30/09/2019

Correspondencia: tbanegasp@ucacue.edu.ec

a. Diploma Superior en Análisis de Datos de Sistemas Complejos; Magister en Gerencia Empresarial, MBA., Mención Gestión de Proyectos; Economista; Técnico en Estadísticas, Económicas y Sociales; Universidad Católica de Cuenca; Cuenca, Ecuador; tbanegasp@ucacue.edu.ec

b. Especialista en Gestión de Proyectos; Magister en Gerencia de Proyectos para el Desarrollo; Magister en Docencia Universitaria y Administración Educativa; Auditor; Contador Público; Ingeniero Empresarial; Tecnólogo Pedagógico en Informática; Universidad Católica de Cuenca; Cuenca, Ecuador; jbecerram@ucacue.edu.ec

c. Magister en Administración de Empresas Mención en Recursos Humanos y Marketing; Especialista en Docencia Universitaria; Contador Público; Ingeniero Comercial; Licenciado en Administración; Universidad Católica de Cuenca; Cuenca, Ecuador; ezamoraz@ucacue.edu.ec

d. Magister en Seguridad Higiene Industrial y Salud Ocupacional; Diploma Superior en Gestión Logística; Magister en Administración de Empresas; Ingeniero Comercial; Técnico Superior en Análisis de Sistemas; Universidad Católica de Cuenca; Cuenca, Ecuador; hespinozap@ucacue.edu.ec 


\section{Los modelos de equilibrio general dinámico estocásticos fundamentación microeconómica}

Vol. 3, núm. 3., (2019)

Tito Max Banegas Peña, Jorge Edison Becerra Molina; Edgar Geovanny Zamora Zamora; Héctor Espinoza Pillaga

\section{RESUMEN}

La investigación llevada a cabo en este artículo fue referente a los modelos de equilibrio general dinámico estocásticos (DSGE): fundamentación microeconómica. El objetivo principal fue el de ver la posición en la cual se encuentran los DSGE, y cuál es el pensamiento que se tiene en el campo de la economía respecto a la fundamentación microeconómica y su aplicación en dichos modelos. Esta se realizó mediante búsqueda y revisión sistema de literatura científico académica seleccionada, la cual fue extraída de las siguientes bases de datos: ResearchGate, SciELO, Redalyc, Scientific Direct y entre otras; todos esto fue parte esencial del proceso de investigación y compresión que ayudo a la síntesis de la evidencia disponible. Como resultado se evidenció que en la economía no se está conforme con los DSGE que se están usando, y muchos economistas acusan que es necesario realizar una reformación de estos, requiriendo hacer un análisis de los fundamentos microeconómicos nuevamente. Finalmente queda como observación que de seguir con los modelos DSGE actuales, es muy probable que la historia se repita, y la economía vuelva a hacer algún movimiento inesperado que lleve naciones enteras a desastres económicos.

Palabras Claves: Macroeconomía; Microeconomía; Equilibrio General Dinámico Estocástico-DSGE; Política Económica. 


\title{
Los modelos de equilibrio general dinámico estocásticos fundamentación \\ microeconómica
}

Vol. 3, núm. 3., (2019)

Tito Max Banegas Peña, Jorge Edison Becerra Molina; Edgar Geovanny Zamora Zamora;

Héctor Espinoza Pillaga

\begin{abstract}
The research carried out in this article was related to stochastic dynamic general equilibrium (DSGE) models: microeconomic foundation. The main objective was to see the position in which the DSGE are, and what is the thinking in the field of economics regarding the microeconomic foundation and its application in these models. This was done through search and review system of selected academic scientific literature, which was extracted from the following databases: ResearchGate, SciELO, Redalyc, Scientific Direct and among others; All this was an essential part of the research and compression process that helped to synthesize the available evidence. As a result, it was evident that the economy is not satisfied with the DSGE that are being used, and many economists accuse that it is necessary to reform them, requiring an analysis of the microeconomic fundamentals again. Finally, it remains as an observation that if we continue with the current DSGE models, history is very likely to repeat itself, and the economy will once again make some unexpected movement that leads entire nations to economic disasters.
\end{abstract}

Key words: Macroeconomics; Microeconomics; Stochastic Dynamic General Balance-DSGE; Economic policy. 


\section{Los modelos de equilibrio general dinámico estocásticos fundamentación microeconómica}

Vol. 3, núm. 3., (2019)

Tito Max Banegas Peña, Jorge Edison Becerra Molina; Edgar Geovanny Zamora Zamora; Héctor Espinoza Pillaga

\section{Introducción.}

La teoría de equilibrio general competitivo se inició con los trabajos de Kenneth Arrow (1951), Gerard Debreu (1973), Arrow y Debreu (1954), McKenzie (1959), Tjalling Koopmans (1957). Sus cimientos datan de escritos del siglo XIX, expuestos por Agustín Cournot (1838), Jules Dupuit (1995), Francis Edgeworth (1881), Alfred Marchall (1890) y León Walras (1874 y 1974). Los trabajos de mediados del siglo XX de Vilfredo Pareto (1909), Gustav Cassel (1932), Jhon Hicks (1939a), Paul Samuelson (1947) Abraham Wald (1934-1935) y John Von Neumann (1937) contribuyeron a la continuación de dichos cimientos (Tello, 2017).

De esta base, se ha desarrollado ramificaciones, como: teoría de juegos (por ejemplo, Von Neumann \& Morgenstern, 1944; Luce \& Raifa, 1957; y Schelling, 1960), teorías del caos o de complejidad (Baumol \& Benhabib, 1989; Brock, 1988; y Grandmont, 1985), modelos de preferencias endógenas (Bowles, 1998) y modelos de información limitada y asimétrica (Stiglitz, 2000). Arrow (1994) afirma que la teoría de equilibrio general es todavía la única forma coherente de entender la economía en su conjunto.

Por otro lado, originado por la contribución de Hurwicz (1960), se ha desarrollado la teoría de diseño de mecanismos económicos, la cual busca o identifica sistemas económicos superiores en términos de objetivos específicos (Hurwicz, 1973). Estos sistemas, tienen las características de ser descentralizados y eficientes económicamente (Hurwincz \& Teiter, 2006). Uno de esos mecanismos es el de mercado base de la teoría de equilibrio general competitivo (Tello, 2017).

Paralelo a estos avances teóricos, los trabajos de Scarf (1967a y 1967b), Scarf y Hansen (1973) y Scarf y Shoven (1984), desarrollaron y popularizaron los denominados modelos aplicados, computables o calibrados de equilibrio general (MCEG). Estas aplicaciones operacionalizan la teoría de equilibrio general para abordar temas relevantes del desarrollo económico de los países. Los MCEG cuantifican los efectos sobre las industrias, ocupaciones regiones al interior de la economía y los grupos socioeconómicos que residen en ella, Los pioneros de los MCEG fueron las numéricas aplicaciones realizadas por Harberger (1962) y Johansen (1960). Anteriormente a estos modelos y en la línea cuantitativa, fueron los trabajos de la matriz insumo-producto y programación lineal realizados por Leontief (1936 y 1941) y Manne (1963).

\section{Definición del equilibrio competitivo}

La Teoría General Competitivo TEGC -en su nivel básico- en una economía abstracta o de carácter esquemático y está conformada por $\mathrm{H}$ individuos o consumidores y $\mathrm{N}$ bienes y/o servicios que serán consumidos por los individuos. Ese bien o servicio tendrá las características de un bien y/o servicio final, o ser usado como un insumo intermedio, para la producción de otros bienes o servicios. Un bien o servicio puede tener ambas características. Una parte de dichos bienes -denominados factores primarios de producción- puede no ser producido por el mercado. En cuyo caso, se asume que, antes del proceso productivo, los consumidores disponen de un número $\mathrm{M}$ de dotaciones fijas de estos factores primarios de producción. Una firma o productor representativo en esta economía -definida por su tecnología de producción- solo tiene el rol de transformar los factores primarios y/o servicios intermedios a productos. Su número, en equilibrio, es indeterminado. Esta no tiene una preferencia y su criterio de decisión es la de maximizar los beneficios económicos, resultante de su actividad productiva. Las decisiones de los agentes (consumidores y dueños de factores de producción y firmas) de esta economía son sobre cantidades y aceptan los precios de mercado, asumiéndolos como fijos. La organización, configuración o estructura de los $\mathrm{N}$ mercados de bienes y/o servicios y factores primarios se asume de competencia perfecta.

Revista Científica Mundo de la Investigación y el Conocimiento. 3 (3). pp. 735-763 


\section{Los modelos de equilibrio general dinámico estocásticos fundamentación microeconómica}

Vol. 3, núm. 3., (2019)

Tito Max Banegas Peña, Jorge Edison Becerra Molina; Edgar Geovanny Zamora Zamora;

Héctor Espinoza Pillaga

Los individuos, consumidores y dueños de los factores primarios y de los beneficios económicos de las firmas buscan su propio interés o bienestar y se interrelacionan en el mercado de esta economía ideal o abstracta. La asignación de recursos resultante del comportamiento de los individuos y firmas y sus interacciones en los mercados es lo que Adam Smith (1759) denominaba como la asignación resultante de la <<mano invisible del mercado〉> sin la necesidad de la intervención del <<Gobierno〉>. En esta economía idealizada no existe gobierno y las decisiones y asignaciones son realizadas en un periodo de tiempo predeterminado (Nuñez, 2012).

\section{Fundamentación teórica.}

Las teorías de la conducta del consumidor asumen que el agente tiene una noción del ingreso que recibirá en un período, así como de los bienes y servicios que comprará, y escoge entre las alternativas de consumo posibles, tal que la satisfacción obtenida de los bienes elegidos sea la mejor; es decir, se valora los bienes con una función de utilidad.

El desarrollo de la teoría de las curvas de indiferencia consolidó el análisis ordinal y ofrece un medio para describir geométricamente la noción de utilidad ordinal, con lo que es posible derivar un modelo de demanda basado en supuestos que son mucho menos rígidos que los que sirven de fundamento al modelo de demanda basado en la utilidad cardinal (Nicholson \& Snyder, 2011).

Este enfoque se limita a que: dado el conjunto de bienes, el consumidor es capaz de decidir si prefiere uno de ellos o si le son indiferentes, es decir; puede definirse una función de utilidad (Nicholson \& Snyder, 2011).

$$
U_{i}=U_{i}\left(x_{i 1}, \ldots, x_{i m}\right)
$$

Donde:

* $U_{i}$ Función creciente de utilidad del consumidor " $i$ ", que depende de las cantidades $x_{i j}$ de cada uno de los " $m$ " bienes de consumo que se producen en la economía.

* $x_{i j}$ Cantidad del bien " $j$ " que consume la unidad " $i$ ".

En el caso básico de dos bienes, la función de utilidad es: $U_{i}=U_{i}\left(x_{i}, y_{i}\right)$, donde $U_{i}$ se mide en forma ordinal, es decir; $(\mathrm{x}, \mathrm{y})$ puede categorizarse de acuerdo con el grado de satisfacción que generan en el consumidor. Dado que $\mathrm{U}_{\mathrm{i}}$ es continua, la igualdad anterior se cumple para un número infinito de combinaciones $(\mathrm{x}, \mathrm{y})$.

Una curva de indiferencia es el lugar geométrico de puntos que son la representación de combinaciones de bienes $(\mathrm{x}, \mathrm{y})$ tales que cualquiera de ellas le es indiferente al consumidor, es decir; indica un nivel de utilidad $\mathrm{U}_{\mathrm{i}}^{0}=\mathrm{U}\left(\mathrm{x}_{\mathrm{i}}, \mathrm{y}_{\mathrm{i}}\right)=\mathrm{C}, \mathrm{C}$ constante.

Un mapa de indiferencia es un conjunto de curvas de indiferencia, cada una de las cuales corresponde a un nivel de satisfacción distinto. En este caso, en lugar de construir una función de utilidad para cada uno de los bienes, se hace una superficie de utilidad, en la que el nivel de utilidad depende simultáneamente de las cantidades consumidas de todos los bienes (Nicholson \& Snyder, 2011). 


\section{Los modelos de equilibrio general dinámico estocásticos fundamentación microeconómica}

Vol. 3, núm. 3., (2019)

Tito Max Banegas Peña, Jorge Edison Becerra Molina; Edgar Geovanny Zamora Zamora; Héctor Espinoza Pillaga

Las curvas de indiferencia poseen propiedades que las caracterizan como:

* Una combinación de bienes ubicados sobre una curva de indiferencia que se encuentra por encima y a la derecha de otra representa una combinación preferida de bienes.

* Las curvas de indiferencia tienen pendiente negativa.

* Las curvas de indiferencia nunca pueden intersectarse.

* Las curvas de indiferencia son convexas hacia el origen.

* Alguna curva de indiferencia pasa por cada uno de los puntos del espacio de bienes.

\section{Optimización del Consumidor}

Si el consumidor desea adquirir la combinación $(x, y)$ cuyo nivel de satisfacción sea el más alto, se obtiene un problema de maximización, pero su ingreso es limitado y no puede adquirir una cantidad ilimitada de productos, entonces la restricción presupuestaria es:

$$
Y=x P_{x}+y P_{y}
$$

Donde:

* $Y$ Es el ingreso del individuo.

* $x$ Es la cantidad consumida del bien $x$.

* $y$ Es la cantidad consumida del bien $y$.

* $P_{x}$ Es el precio del bien $x$.

* $P_{y}$ Es el precio del bien $y$.

El problema del consumidor consiste en elegir la combinación $(x, y)$ que logre el más alto grado de satisfacción sin sobrepasar su presupuesto. Los factores que influyen en el consumidor son el precio de ambos bienes y su ingreso.

Con precios e ingreso constantes, la línea $A B$ de la Figura (1) representa todas las combinaciones posibles de bienes que el consumidor puede obtener con su ingreso, la cual es la representación gráfica de la ecuación $(2,1)$, cuando $Y$ es constante (Nicholson \& Snyder, 2011).

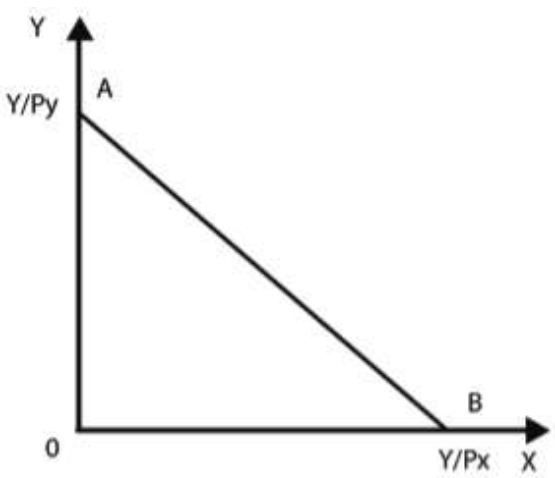

Figura 1: Restricción presupuestaria

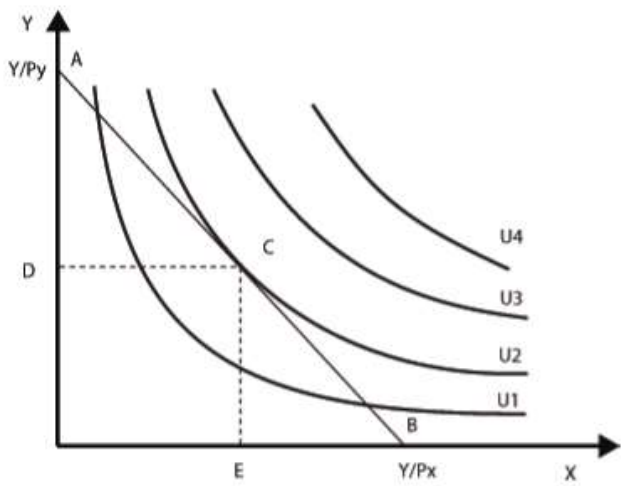

Figura 2: Restricción presupuestaria 


\section{Los modelos de equilibrio general dinámico estocásticos fundamentación microeconómica}

Vol. 3, núm. 3., (2019)

Tito Max Banegas Peña, Jorge Edison Becerra Molina; Edgar Geovanny Zamora Zamora; Héctor Espinoza Pillaga

Su pendiente depende del precio de los bienes, así se obtiene: $y=\frac{Y}{P_{y}}-x \frac{P_{x}}{P_{y}}$ que indica que el eje de las $y$ es intersectado en $\frac{Y}{P_{y}}$ y que la pendiente de la línea es $\frac{-P_{x}}{P_{y}}$. Si colocamos la ecuación en función de $y$, tendríamos la intersección sobre el eje $x$, sin variar la pendiente.

La solución al problema de la maximización se logra superponiendo las curvas de indiferencia sobre la recta presupuestaria (Figura 2). El consumidor querrá situarse en la curva de indiferencia más elevada que pudiera alcanzar con su presupuesto $A B$ que es tangente a la curva de indiferencia $U_{2}$ (Punto $C$ ) que es el punto de máxima satisfacción.

Matemáticamente, el problema anterior se expresa de la siguiente manera:

$$
\left\{\begin{array}{l}
\text { máx } U(x, y) \\
\text { Sujeto } a: \\
Y=x P_{x}+y P_{y} \\
x \geq 0, \quad y \geq 0
\end{array}\right.
$$

Para la maximización de la función $\mathrm{U}=\mathrm{U}(\mathrm{x}, \mathrm{y})$ se usa el método de Multiplicadores de Lagrange, para lo cual se construye el Lagrangeano $\mathcal{L}$ :

$$
\mathcal{L}=U(x, y)+\mathcal{L} *\left(Y-x P_{x}-y P_{y}\right)
$$

Obtener el máximo de $\mathcal{L}$ es equivalente a obtener el máximo de $\mathrm{U}(\mathrm{x}, \mathrm{y})$ sujeta a la condición (1a). Además, $\mathrm{U}(\mathrm{x}, \mathrm{y})$ solo es idénticamente igual a $\mathcal{L}$, para aquellos valores de las variables que satisfacen dicha restricción.

Se asume que la función U es estrictamente cóncava y que el conjunto de soluciones factibles es convexo; por lo tanto, sólo necesitamos examinar condiciones de primer orden que exigen que las primeras derivadas parciales de $\mathcal{L}$ se anulen para máximos y mínimos. Como se aprecia, es un sistema de tres ecuaciones con tres incógnitas: $\mathrm{x}, \mathrm{y}, \lambda$.

$$
\begin{aligned}
& \frac{\partial \mathcal{L}}{\partial x}=\frac{\partial U(x, y)}{\partial x}-\lambda P_{x}=0 \\
& \frac{\partial \mathcal{L}}{\partial y}=\frac{\partial U(x, y)}{\partial y}-\lambda P_{y}=0 \\
& \frac{\partial \mathcal{L}}{\partial \lambda}=Y-x P_{x}-y P_{y}=0
\end{aligned}
$$

De $(2,2)$ se deduce la condición de primer orden 


\section{Los modelos de equilibrio general dinámico estocásticos fundamentación microeconómica}

Vol. 3, núm. 3., (2019)

Tito Max Banegas Peña, Jorge Edison Becerra Molina; Edgar Geovanny Zamora Zamora; Héctor Espinoza Pillaga

$$
\lambda=\frac{\frac{\partial U(x, y)}{\partial x}}{P_{x}}=\frac{\frac{\partial U(x, y)}{\partial y}}{P_{y}} \Rightarrow \frac{\frac{\partial U(x, y)}{\partial x}}{\frac{\partial U(x, y)}{\partial y}}=\frac{P_{x}}{P_{y}} ; \text { es decir: } \frac{U_{m g x}}{U_{m g y}}=\frac{P_{x}}{P_{y}}
$$

El cociente de las utilidades marginales indica la pendiente de la curva de indiferencia mientras que el cociente de los precios es la pendiente de la recta de presupuesto. Lambda, $\lambda=\frac{\partial U}{\partial Y}$ mide la variación de la utilidad debida a una variación en el ingreso (Nicholson \& Snyder, 2011).

Las condiciones de segundo orden dan criterios de puntos máximos o mínimos locales, dependiendo si la Matriz Hessiana es definida positiva o negativa.

$$
H=\left(\begin{array}{ccc}
\frac{\partial^{2} U(x, y)}{\partial x^{2}} & \frac{\partial U(x, y)}{\partial y} & -P_{x} \\
\frac{\partial U(x, y)}{\partial x} & \frac{\partial^{2} U(x, y)}{\partial x^{2}} & -P_{y} \\
-P_{x} & -P_{y} & 0
\end{array}\right) \geq 0 ; \text { sí: } x^{T} H_{x} \geq 0, \text { ó, } x^{T} H_{x}<0 \quad \forall x \in \mathbb{R}^{2}
$$

Ya que el ingreso del consumidor y los precios de los productos son conocidos, se pueden determinar las cantidades de cada producto requeridas, es decir; la solución $\left(x^{*}, y^{*}\right)$ que está en términos de los parámetros $P_{x}, P_{y}$ e $Y$ son las funciones de demanda, con una función de utilidad explícita y junto con el análisis de las curvas de indiferencia se deduce la curva de demanda por bien.

\section{La demanda y función de demanda}

La deducción de la curva de demanda admite un análisis gráfico, para lo cual se asume variaciones del precio de la canasta $(x, y)$. Dados $P_{x_{1}}$ y $P_{y_{3}}$ iniciales, el consumidor está en equilibrio y consume $x_{1}$ e $y_{3}$ (Figura 3). Ahora, el problema es establecer las cantidades de $x$ que se comprarán cuando varíe $P_{x}$, estando en equilibrio en cada uno de esos precios (Nicholson \& Snyder, 2011). 


\section{Los modelos de equilibrio general dinámico estocásticos fundamentación microeconómica}

Vol. 3, núm. 3., (2019)

Tito Max Banegas Peña, Jorge Edison Becerra Molina; Edgar Geovanny Zamora Zamora; Héctor Espinoza Pillaga

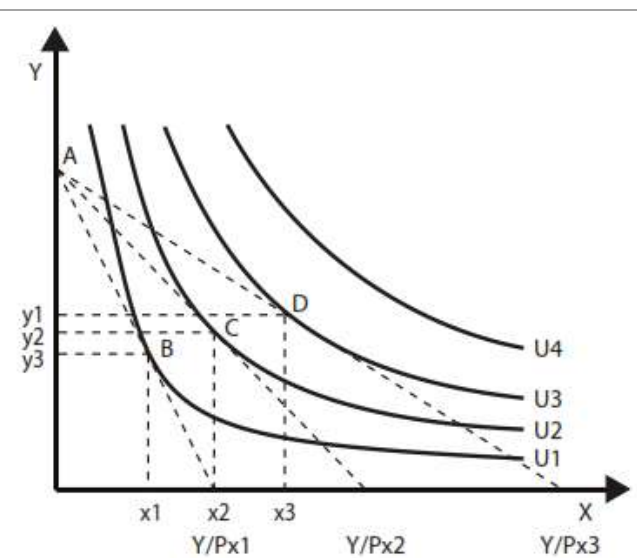

Figura 3: Restricción presupuestaria

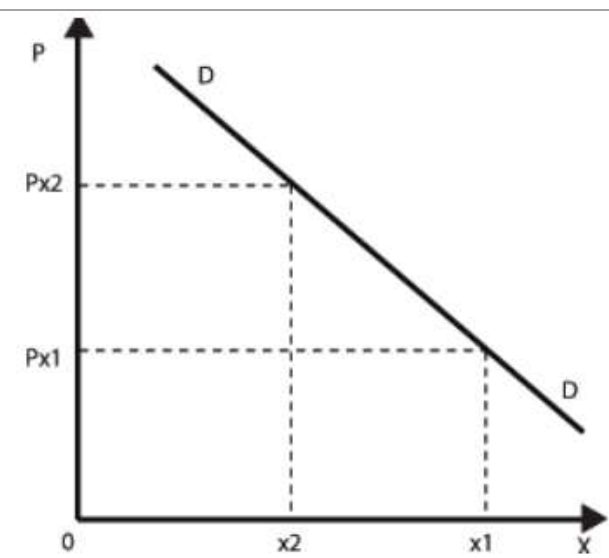

Figura 4: Curva de demanda

Si el precio de bien $x$ se eleva de $P_{x_{1}}$ a $P_{x_{2}}$ y si el nivel de consumo se mantiene, $\frac{\partial U(x, y)}{\partial x}$ permanece invariable, pero $\left(\frac{U m g x 1}{P_{x_{2}}}\right)$ habrá disminuido. Si al precio $P_{x_{2}}$ se continúa consumiendo la cantidad $x_{1}$, se gastará una parte mayor de su ingreso en el bien $x$, dejando menos ingreso para gastar en $y$.

Como el ingreso $Y$ y el precio $p_{Y}$ permanecen constantes, necesariamente se comprará menos de $y$. La disminución del número de unidades consumidas de $y$ hace que $\frac{\partial U(x, y)}{P_{x}}$ se incremente, incrementando $\frac{U m g y}{P_{y}}$, violándose la condición de máxima utilidad.

$$
\frac{U m g x}{P_{x}}<\frac{U m g y}{P_{y}}
$$

Por lo tanto, se transferirá gasto del bien $\mathrm{x}$ al bien $\mathrm{y}$ hasta hallar su nuevo punto de equilibrio. Cada uno de estos puntos de equilibrio determina la cantidad demandada de cada bien determinando sus curvas de demandas, que generalmente, serán decrecientes (Figura 4).

De lo anterior se deducen dos propiedades importantes

1. La demanda de cualquier producto es función unívoca de los precios y el ingreso.

2. Las funciones de demanda son homogéneas de grado cero en precios e ingreso, es decir: si todos los precios y el ingreso varían en igual valor, las cantidades demandadas permanecen invariables.

La primera propiedad proviene de la convexidad de las curvas de indiferencia: a una serie dada de precios y rentas corresponde un solo máximo y por lo tanto una sola canasta $(\mathrm{x}, \mathrm{y})$.

En la segunda propiedad, se asume que los precios e ingreso varían en un valor $\mathrm{K}$, es decir; $\mathrm{K} * \mathrm{Y}=$ $\mathrm{x}\left(\mathrm{K} * \mathrm{P}_{\mathrm{x}}\right)+\mathrm{y}\left(\mathrm{K} * \mathrm{P}_{\mathrm{y}}\right)$, la cual no afecta al óptimo. Esto significa que, en términos de ingreso real, el consumidor no se comportará como si fuese más rico (o más pobre), estos cambios no alteran su conducta (Nicholson \& Snyder, 2011). 


\section{Los modelos de equilibrio general dinámico estocásticos fundamentación microeconómica}

Vol. 3, núm. 3., (2019)

Tito Max Banegas Peña, Jorge Edison Becerra Molina; Edgar Geovanny Zamora Zamora; Héctor Espinoza Pillaga

La demanda individual de un bien se define a través de: el precio del bien, el ingreso del consumidor, el precio de los demás bienes, de la cantidad comprada, así como de los gustos y preferencias del consumidor. La ecuación de demanda de un bien llamado " $\mathrm{x}$ ", puede escribirse entonces como: $\mathrm{X}=\mathrm{f}\left(\mathrm{P}_{\mathrm{x}}\right)$. Matemáticamente:

$$
q_{i}=f_{i}\left(p q_{1}, p q_{2}, p q_{3}, \ldots, Y, G\right)
$$

Donde:
* $q_{i}$ : Cantidad demandada del bien.
* $p q_{1}$ : Precio del bien $q_{1}$ :
* $p q_{i}$ : Precio de todos los demás bienes, $i=2 \ldots m$.
* Y: Nivel de ingreso.
* G: Gustos y preferencias del consumidor.

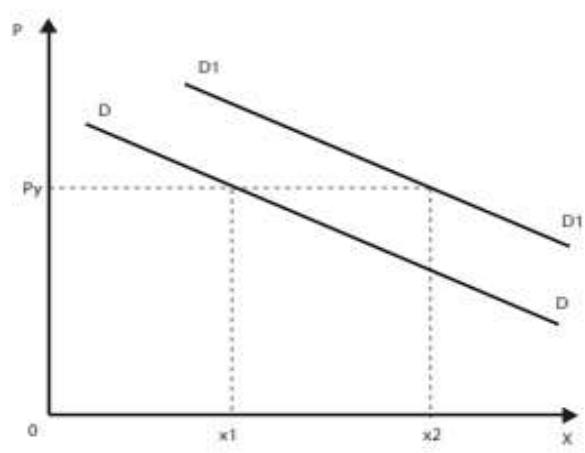

Figura 5: Curva de demanda

Un movimiento a lo largo de la curva de demanda consiste en un cambio en la cantidad comprada como resultado de un cambio en el precio del bien. Si cambia alguno de los otros factores condicionantes, la curva cambiará, así un aumento en los ingresos de los consumidores llevará la curva, desde DD hasta $\mathrm{D}_{1} \mathrm{D}_{1}$ (Figura 5).

Con ingresos mayores los consumidores desearán aumentar sus compras a cada precio (bienes normales). El efecto de cambio en los precios de bienes relacionados sobre la demanda del bien $x$ depende de la naturaleza de la relación que los ligue, lo cual se estudia usando el concepto de elasticidades (Nicholson \& Snyder, 2011).

La curva de demanda de mercado se compone de las curvas de demandas individuales. La misma se define a suma horizontal de las curvas de demanda individuales.

La reacción del consumidor ante cambios de los precios e ingreso se analiza a través del concepto de elasticidad.

$>$ La elasticidad precio mide el cambio porcentual que se produce en la cantidad demandada ante un cambio porcentual unitario del precio del bien.

$>$ La elasticidad ingreso mide la misma reacción, pero ante cambios en el ingreso del consumidor.

$>$ La elasticidad cruzada mide los cambios en la cantidad demandada de un bien ante cambios en los precios de otros bienes.

\section{Estudio de la Oferta}

El análisis de la empresa es similar al análisis del consumidor, este adquiere artículos con los que produce satisfacción dada una función de utilidad, mientras que la empresa adquiere insumos con los que produce artículos dada una función de producción. La ecuación de ingreso del consumidor es función lineal de las cantidades de artículos que compra y la ecuación de costo de la empresa es una función lineal de las cantidades de insumos que adquiere (Nicholson \& Snyder, 2011). 


\section{Los modelos de equilibrio general dinámico estocásticos fundamentación microeconómica}

Vol. 3, núm. 3., (2019)

Tito Max Banegas Peña, Jorge Edison Becerra Molina; Edgar Geovanny Zamora Zamora;

Héctor Espinoza Pillaga

La función de utilidad es subjetiva, la utilidad no tiene una medición cardinal concreta, mientras que la función de producción es objetiva y el producto de la empresa es medible. El consumidor racional maximiza su utilidad con un ingreso dado, lo análogo del empresario es maximizar su producto con un costo dado (fijo o variable), deseando maximizar su beneficio.

\section{La Función de Producción}

El término "función de producción" se refiere a la relación física entre los recursos de una firma y su producción (bienes o servicios) por período sin considerar los precios. La producción de bienes se representa por $Q_{i}$ y los insumos por $v_{i}$ :

$$
Q_{i}=f_{i}\left(v_{1}, \ldots, v_{n}\right)
$$

La función de producción establece el máximo producto obtenible de cada posible combinación de insumos, la mejor utilización de cada combinación de insumos no es un problema económico si no técnico y la selección de la mejor combinación de insumos para la producción de un nivel de producto depende de los precios y es objeto del análisis económico (Nicholson \& Snyder, 2011).

\section{Curvas de productividad}

Para dos insumos $\mathrm{v}$ y $\mathrm{w}$, la productividad de $\mathrm{v}$ en $\mathrm{Q}$ se define como la cantidad de $\mathrm{Q}$ que puede obtenerse del insumo $v$, manteniendo fijo $\mathrm{w}$, o sea $\mathrm{Q}=\mathrm{f}\left(\mathrm{v}, \mathrm{w}_{0}\right)$. Cada curva relaciona $\mathrm{Q}$ y $\mathrm{v}$ al variar $\mathrm{w}_{0}$ originando las curvas de productividad total (Figura 6). Un aumento de $\mathrm{w}_{0}$ reducirá la cantidad de $\mathrm{v}$ necesaria para producir cada nivel de producto.

La productividad media $(P M)$ del insumo $v$ es su productividad total sobre su cantidad.

$$
\begin{gathered}
P M=\frac{Q}{v}=\frac{f\left(v, w_{0}\right)}{v} \\
P m g v=\frac{\partial Q}{\partial v}=f^{\prime}(v)
\end{gathered}
$$

La productividad marginal $(P m g)$ del insumo $v$, es la relación entre las variaciones de su productividad total y las variaciones en su cantidad.

En la (Figura 7) se representan las curvas de $P M$ y $P m g$ correspondiente a una de las curvas de productividad total. La curva de $P m g$ alcanza un máximo a un nivel de insumo inferior al que determina el máximo de la curva $P M$ y corta a $P M$ en su punto máximo (Nicholson $\&$ Snyder, 2011). 


\section{Los modelos de equilibrio general dinámico estocásticos fundamentación microeconómica}

Vol. 3, núm. 3., (2019)

Tito Max Banegas Peña, Jorge Edison Becerra Molina; Edgar Geovanny Zamora Zamora; Héctor Espinoza Pillaga

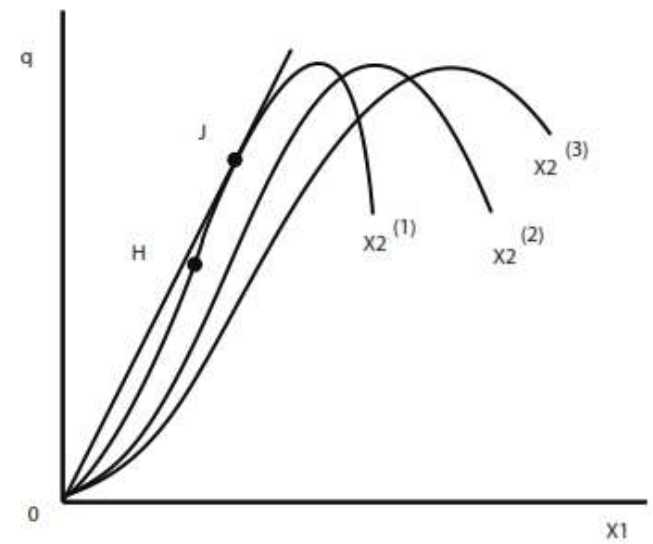

Figura 6: Productividad total

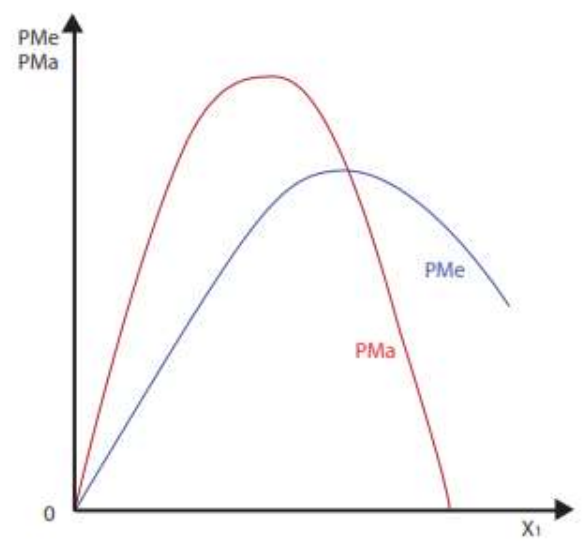

Figura 7: PM y Pmg

Si $P M$ está en un extremo se tiene:

$$
\frac{d}{d v}(P M)=0=\frac{d}{d v}\left(\frac{Q(v)}{v}\right)=\frac{\frac{\partial Q}{\partial v} * v-Q}{v^{2}} \Leftrightarrow \frac{\partial Q}{\partial v} * v-Q=0 \Leftrightarrow \frac{\partial Q}{\partial v}=\frac{Q}{v}
$$

Lo cual determina, que en el punto donde la $P M$ se hace máxima iguala a la $P m g$, punto para el que la pendiente de su tangente es igual a cero.

El nivel de insumo para el que la Pmg alcanza un máximo, es el mismo que el del punto de inflexión de la curva correspondiente de productividad total (punto $H$ de la curva $x_{2}$, figura 6). El nivel de insumo en que la curva $P M$ alcanza un máximo, es el mismo nivel de insumo para el que la pendiente del vector que va desde el origen hasta la curva de productividad total, alcanza un máximo (punto $J$ de la curva $x_{2}$ figura 7) (Criollo, 2010).

\section{Isocuantas}

Una isocuanta es en la teoría de la producción, lo que la curva de indiferencia representa para el consumidor, es el lugar geométrico de todas las combinaciones de factores $(v, w)$ que proporcionan un nivel de producto específico, para un nivel dado de producto se tiene que la función de producción será: $Q_{0}=f(v, w)$.

La pendiente de la tangente a una isocuanta, es la relación a la que debe cambiarse $v$ por $w$ para mantener constante el nivel de producto denominada Razón Marginal de Sustitución Técnica (RMTS), un concepto análogo al de tasa marginal de sustitución del consumidor.

Las cantidades óptimas de uso de insumos se obtienen con el análisis de la productividad media y marginal de cada uno de los insumos y con este análisis, se obtiene interpretaciones adecuadas de los niveles de producción que deberían tenerse (Barreiro, Labeage, \& Mochón, 1999). 


\section{Los modelos de equilibrio general dinámico estocásticos fundamentación microeconómica}

Vol. 3, núm. 3., (2019)

Tito Max Banegas Peña, Jorge Edison Becerra Molina; Edgar Geovanny Zamora Zamora; Héctor Espinoza Pillaga

El diferencial total de la función de producción es: $d Q=\frac{Q d v}{v}+\frac{Q d w}{w}$, donde $\frac{\partial Q}{\partial v}$ y $\frac{\partial Q}{\partial w} \quad$ son las

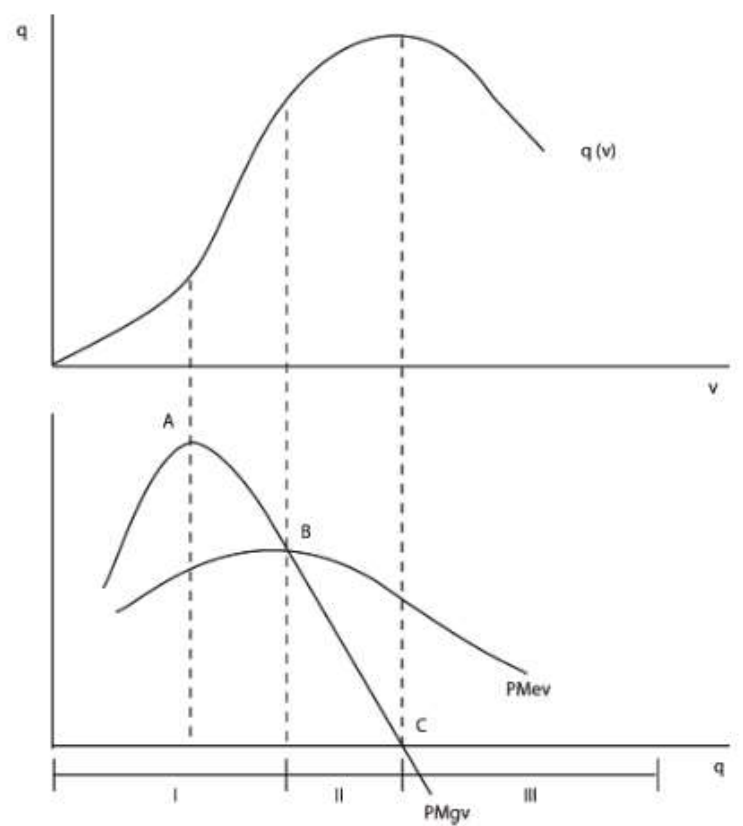

Figura 8: Producción, PMe y Pmg productividades marginales de $v$ y $w$. Como $d Q=0$ para movimientos a lo largo de una isocuanta $(P m g v) d v+(P m g w) d w=0, \quad$ entonces $R M T S=\frac{d v}{d w}=-\frac{P m g w}{P m g v}$

En el punto $A$ se obtiene la máxima productividad marginal del insumo $v$, en el punto $B$, la producción media de $v$ es igual a la producción marginal de $v$ y en el punto $C$ el producto marginal de $v$ es cero. El empresario racional nunca utilizará un factor de producción tal que su productividad marginal sea negativa $P m g v<0$.

Las isocuantas miden las diferentes escalas de producción, es decir los diferentes niveles de producto ante incrementos en todos los insumos de la producción. La forma en cómo se desplazan las isocuantas ante variaciones en la combinación de insumos se define como los rendimientos a escala pudiendo ser los mismos constantes, crecientes o decrecientes.

Los mapas de isocuantas que aparecen en la siguiente figura muestran: rendimientos constantes a escala, rendimientos crecientes a escala y rendimientos decrecientes a escala, respectivamente, para dos factores de producción: Trabajo $(L)$ y Capital $(K)$.

\section{Isocostos}

Si se compra los insumos $v$ y $w$ a precios unitarios constantes $r_{v}$ y $r_{w}$ respectivamente, el costo total de producción es: $C=r_{v} * v+r_{w} * w$. El lugar geométrico de las combinaciones de insumos que pueden comprarse a un costo total $C_{0}$, se denomina línea de isocosto $C_{0}=r_{v} * v+r_{w} * w$

La pendiente de la línea de isocosto es igual a la razón de los precios de los insumos con signo negativo. $\mathrm{Si}$ mayor es el gasto total de una línea isocosto, mayores son los segmentos limitados por las intersecciones sobre los ejes $v$ y $w$, por lo tanto, más alejada se encuentra del origen: CT3 $>$ CT2 > CT1. 


\section{Los modelos de equilibrio general dinámico estocásticos fundamentación microeconómica}

Vol. 3, núm. 3., (2019)

Tito Max Banegas Peña, Jorge Edison Becerra Molina; Edgar Geovanny Zamora Zamora; Héctor Espinoza Pillaga

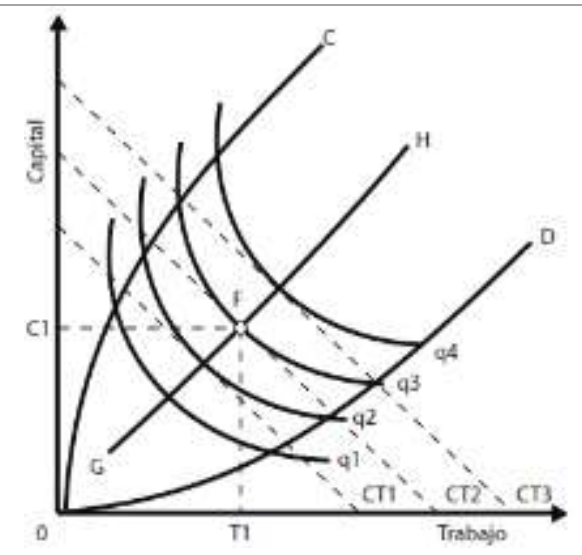

Figura 9: Isocostos

La óptima combinación de insumos viene dada por el punto de tangencia entre una isocuanta y la línea de isocosto pertinente. Si $C_{1}$ es el nivel predeterminado de costo (figura 9) el producto máximo será $q_{3}$. Estos puntos de tangencia determinan la senda de expansión (Nicholson \& Snyder, 2011).

\section{Optimización del Productor}

El empresario quiere obtener el mayor producto posible con un costo dado, con lo que se obtiene un problema de optimización muy similar al de Maximización de la Utilidad del Consumidor, es decir: se debe maximizar la función:

$$
V=f(v, w)+\mu *\left(C_{0}-r_{v} * v-r_{w} * w\right)
$$

Con condiciones de primer orden (CPO):

$$
\begin{gathered}
\frac{\partial V}{\partial v}=\frac{\partial f(v, w)}{\partial v}-\mu *\left(r_{v}\right)=0 \\
\frac{\partial V}{\partial w}=\frac{\partial f(v, w)}{\partial w}-\mu *\left(r_{w}\right)=0 \\
\frac{\partial V}{\partial \mu}=C_{0}-r_{v} * v-r_{w} * w=0 \\
\frac{\frac{\partial f(v, w)}{\partial v}}{\frac{\partial f(v, w)}{\partial w}}=\frac{r_{v}}{r_{w}}=\frac{P m g v}{P m g w} \Leftrightarrow \mu=\frac{P m g v}{r_{v}}=\frac{P m g w}{r_{w}}
\end{gathered}
$$

Es decir, la contribución del último dólar gastado en cada insumo debe ser igual para todos los insumos e igual a $\mu$, donde $\mu$ (Multiplicador de Lagrange) es la derivada total del producto con respecto al costo, cuando los precios son constantes y las cantidades variables (Nicholson \& Snyder, 2011). 


\section{Los modelos de equilibrio general dinámico estocásticos fundamentación \\ microeconómica}

Vol. 3, núm. 3., (2019)

Tito Max Banegas Peña, Jorge Edison Becerra Molina; Edgar Geovanny Zamora Zamora;

Héctor Espinoza Pillaga

En efecto, suponiendo que el costo es variable, el diferencial total de la ecuación de costo es:

$$
d C=\left(r_{v}\right) d v+\left(r_{w}\right) d w \text { ó } d C=\frac{1}{\mu}\left(\frac{\partial f(v, w)}{\partial v} d v+\frac{\partial f(v, w)}{\partial w} d w\right) \operatorname{con} r_{v}=\frac{\frac{\partial f(v, w)}{\partial v}}{\mu}, \quad r_{w}=\frac{\frac{\partial f(v, w)}{\partial w}}{\mu}
$$

El diferencial total de la ecuación para $Q$ es:

$$
d Q=\frac{\partial f(v, w)}{\partial v} d v+\frac{\partial f(v, w)}{\partial w} d w
$$

Obteniendo:

$$
\frac{d Q}{d C}=\mu \frac{\frac{\partial f(v, w)}{\partial v} d v+\frac{\partial f(v, w)}{\partial v} d w}{r_{v} d v+r_{w} d w}=\mu
$$

\section{Maximización del Beneficio}

El empresario tiene libertad para variar los niveles de costo-producto y su objetivo es la maximización del beneficio, no la solución de máximos o mínimos condicionados. Su ingreso es igual al número de unidades que vende por el precio unitario $p$ conocido. Su beneficio $B(v, w)$ es la diferencia entre el ingreso total y el costo total (Nicholson \& Snyder, 2011).

$$
\begin{aligned}
& B=p Q-c(Q) \\
& B=p \frac{\partial f(v, w)}{\partial v}-\left(r_{v}\right) v-\left(r_{w}\right) w
\end{aligned}
$$

Las condiciones de primer orden:

$$
\begin{aligned}
& \frac{\partial B}{\partial v}=p \frac{\partial f(v, w)}{\partial v}-r v=0 \quad \Rightarrow \quad p \cdot(P m g v)=r v \\
& \frac{\partial B}{\partial w}=p \frac{\partial f(v, w)}{\partial v}-r w=0 \quad \Rightarrow \quad p .(P m g v)=r v
\end{aligned}
$$

El producto P. Pmgv es el valor de la productividad marginal del insumo $v$, y mide la relación a la que aumentaría el ingreso del empresario cuando se incrementa en una unidad la cantidad del insumo $v$. El empresario puede aumentar su beneficio empleando unidades adicionales de $v$ que incrementan el ingreso y cubren los costos adicionales. 


\section{Los modelos de equilibrio general dinámico estocásticos fundamentación microeconómica}

Vol. 3, núm. 3., (2019)

Tito Max Banegas Peña, Jorge Edison Becerra Molina; Edgar Geovanny Zamora Zamora; Héctor Espinoza Pillaga

\section{Metodología.}

El tipo de investigación está ligado con el diseño de campo y el nivel descriptivo, en cuanto se analizaron los procesos, basados en el método de los modelos de equilibrio general aplicado (MEGA).

El MEGA proporciona una simulación de laboratorio empírica y versátil para analizar cuantitativamente los efectos de las políticas económicas, tales como la liberalización comercial y los impactos exógenos del país sujeto de estudio.

La matriz de contabilidad social (MCS), proporciona el marco conceptual que enlaza a los diferentes componentes del modelo y proporciona también gran parte de los datos requeridos en el análisis. Comúnmente para el modelo MEGA se utiliza el programa o software GAMS (General Algebraic Modeling Systern), que se basa en un modelo estadunidense (Robinson, Kilkenny y Hanson, 1990).

\section{Resultados.}

\section{Análisis del circuito económico}

Un sistema económico basado en la división del trabajo y el intercambio se caracteriza por la continua circulación de recursos reales (bienes y servicios) y de recursos financieros (medios de pago) entre los agentes que conforman dicho sistema. La idea del circuito tiene origen en el esfuerzo de describir el complejo proceso de generación y apropiación del ingreso y la riqueza de un país (Algoritmos et al., 2006).

Una forma de representar el circuito económico es considerar dos tipos de agentes, los empresarios y los trabajadores; los primeros compran la fuerza de trabajo proporcionada por los trabajadores, producen bienes y servicios destinados a la venta. Y los trabajadores, formada por quienes venden su fuerza de trabajo a cambio de un salario con el que consumen (Algoritmos et al., 2006). 


\section{Los modelos de equilibrio general dinámico estocásticos fundamentación \\ microeconómica}

Vol. 3, núm. 3., (2019)

Tito Max Banegas Peña, Jorge Edison Becerra Molina; Edgar Geovanny Zamora Zamora;

Héctor Espinoza Pillaga

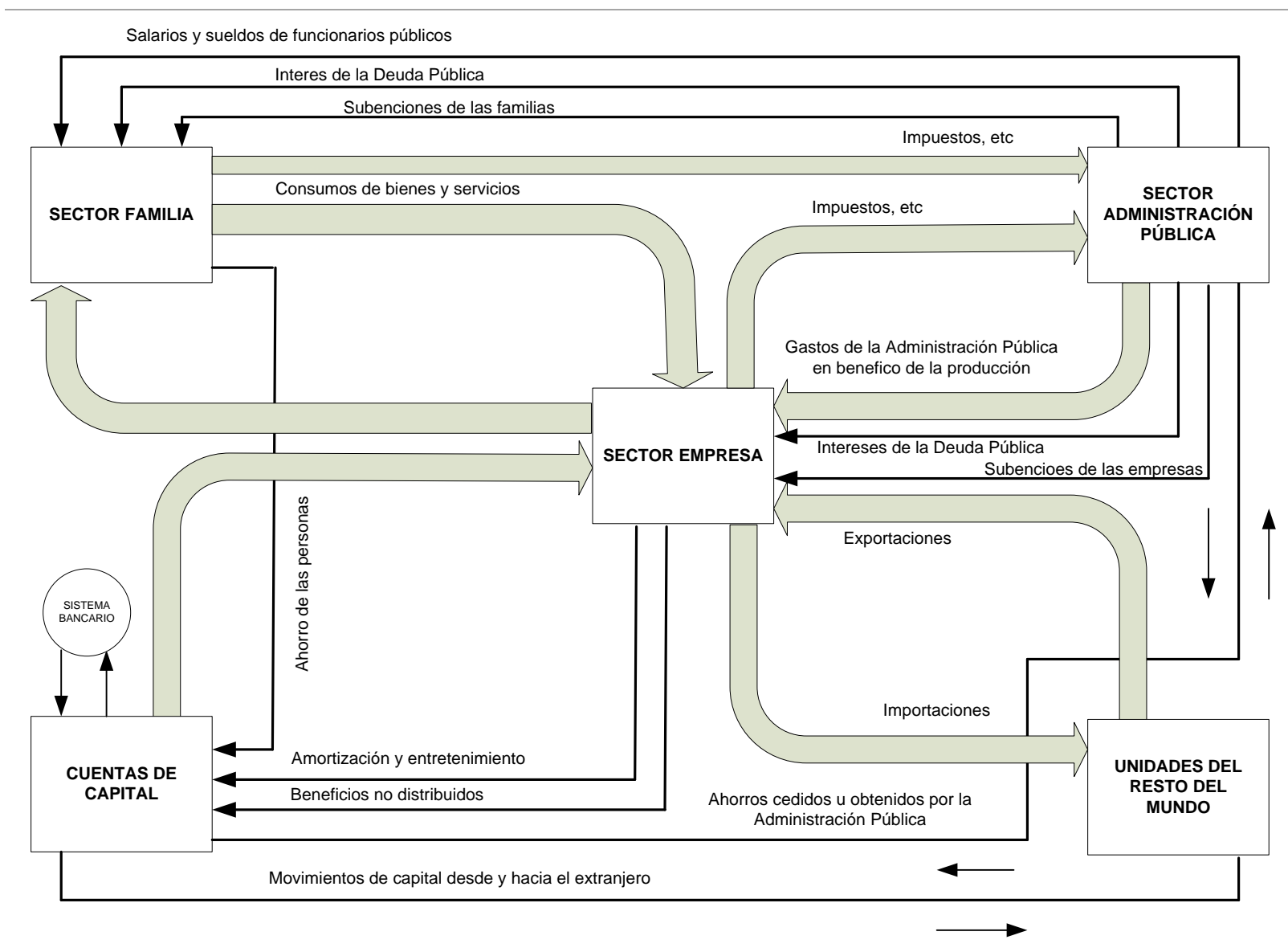

Figura 10: Circuito Económico`

En términos generales el circuito económico considera un flujo real y monetario, cuyos registros usan el principio de contabilidad general y de partida doble.

Las familias necesitan ganar ingresos, para lo cual venden su trabajo a las empresas o al gobierno, con lo cual obtienen recursos y satisfacen necesidades adquiriendo bienes y servicios que compran a las empresas; además deben pagar impuestos al gobierno a cambio de los servicios que éste presta. Por otra parte, las familias, son dueñas de las empresas y reciben las utilidades (o pagan las pérdidas) que las empresas generen.

Las empresas necesitan mano de obra para producir, a la vez que venden sus productos a los hogares y al gobierno, también pagan impuestos al gobierno a cambio de los servicios que recibe, usarán los mínimos recursos posibles para producir, con el fin de poder vender sus bienes y servicios más baratos, ganar a la competencia y maximizar el beneficio (Algoritmos et al., 2006). 


\section{Los modelos de equilibrio general dinámico estocásticos fundamentación microeconómica}

Vol. 3, núm. 3., (2019)

Tito Max Banegas Peña, Jorge Edison Becerra Molina; Edgar Geovanny Zamora Zamora; Héctor Espinoza Pillaga

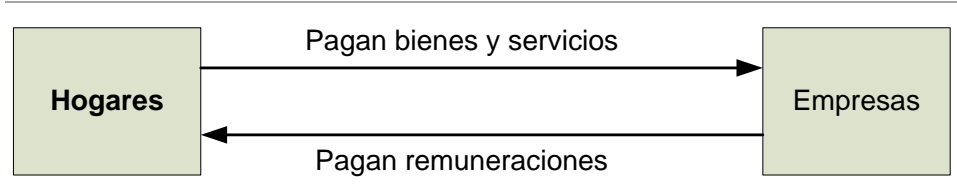

Las relaciones entre los hogares y el gobierno se basan en el pago de impuestos al gobierno, que consume mano de obra de los hogares y les entrega servicios, hace transferencias y da subsidios, sin embargo, el gobierno no tiene los mismos estímulos que las empresas para producir con los recursos que dispone, ya que no tiene que competir con nadie.

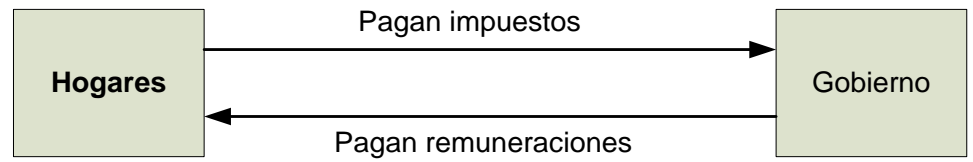

Las relaciones entre empresas y gobierno se describen cuando el gobierno compra productos y servicios de las empresas, a su vez el gobierno maneja aranceles de las materias primas que éstas usan en sus procesos productivos y de los productos que exportan, además provee servicios básicos para que produzcan. Las empresas pagan impuestos al gobierno por todos los servicios, ganancias y respaldo que este aporta al sector privado (Algoritmos et al., 2006).

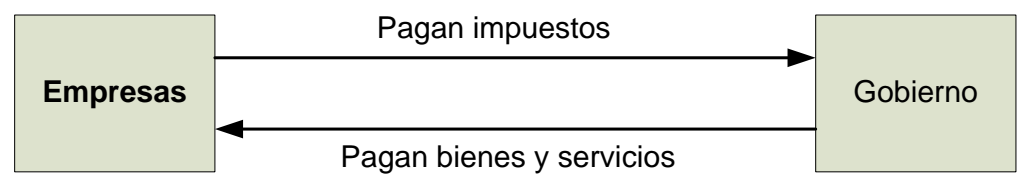

\section{La Matriz de Contabilidad Social MCS y los Modelos MEGA}

Una MCS es la síntesis de dos ideas bien conocidas en economía. La primera se deriva del cuadro insumo-producto y describe el sistema de vínculos inter industriales en la economía. La segunda idea incluida en la MCS, derivada de la contabilidad del ingreso nacional, es que el ingreso siempre es igual al gasto (Pedauga, Sáínez, \& Velázquez, 2012).

La MCS también distingue entre "actividades" y "mercancías", logrando dos efectos diferentes. En primer lugar, permite más de un solo tipo de actividad para producir la misma mercancía, considerando así tecnologías diferentes de producción. En segundo lugar, este tratamiento resuelve problemas difíciles que surgen al tener que manejar importaciones. Si éstas son, de algún modo competitivas con los bienes de producción doméstica (lo que generalmente ocurre), entonces la demanda doméstica estará armada por los dos tipos de bienes. 


\section{Los modelos de equilibrio general dinámico estocásticos fundamentación microeconómica}

Vol. 3, núm. 3., (2019)

Tito Max Banegas Peña, Jorge Edison Becerra Molina; Edgar Geovanny Zamora Zamora;

Héctor Espinoza Pillaga

\section{Cuadro 1}

Esquema de una Matriz de Contabilidad Social

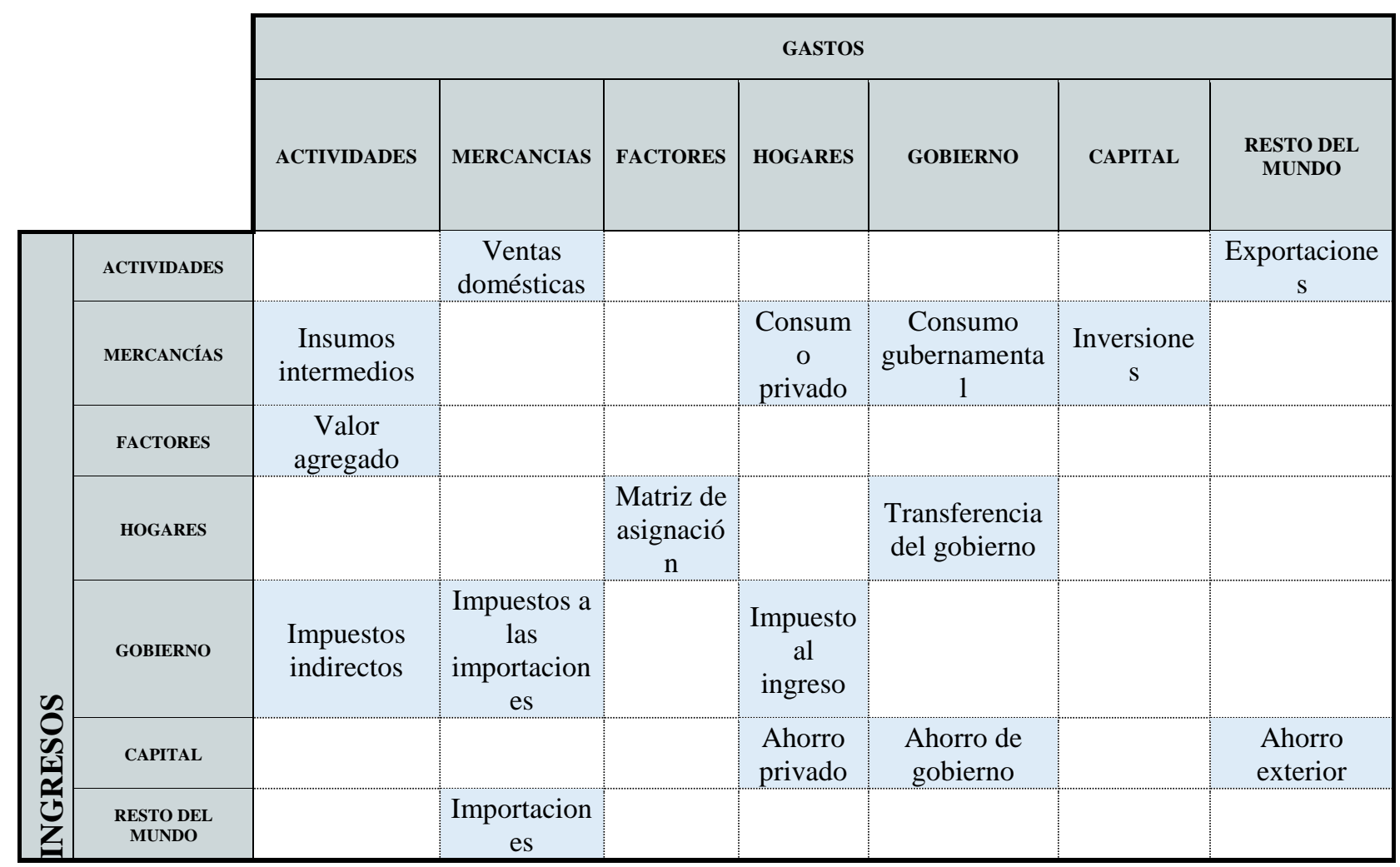

Fuente: Banco Central del Ecuador, (2016)

Elaborado: Autores

La especificación de un modelo "completo" requiere que las relaciones de mercado, de comportamiento y de sistema incluidas en cada cuenta de la MCS sean descritas en el modelo. Las cuentas para actividades, mercancías y factores requieren todas ellas la especificación del comportamiento del mercado (oferta, demanda y condiciones para, el vaciado de los mercados). Las cuentas para hogares y gobierno incluyen las restricciones presupuestales de las familias y del sector público (ingreso igual a gasto). Finalmente, las cuentas para capital y resto del mundo representan los requerimientos macroeconómicos para el balance interno (ahorro igual a inversión) y externo (exportaciones más entradas de capital igual a importaciones) (Haro, 2008).

\section{Ecuaciones del Modelo Base MEGA}

La MCS proporciona una descripción esquemática del flujo circular del ingreso en la economía: desde actividades y mercancías a factores de producción, hasta la llegada a instituciones y de vuelta a actividades y mercancías. La exposición de las ecuaciones del modelo base MEGA sigue el mismo patrón de generación de ingreso. Primero presentamos ecuaciones que definen el sistema de precios, seguidas por ecuaciones que describen la generación de la producción y del valor agregado. A continuación, se presentan ecuaciones que describen el mapeo del valor agregado hacia el ingreso institucional -o de los hogares. El flujo circular se completa entonces mediante ecuaciones que muestran el balance entre la oferta y la demanda de bienes por parte de los varios actores del sistema económico. Finalmente, existe 


\section{Los modelos de equilibrio general dinámico estocásticos fundamentación microeconómica}

Vol. 3, núm. 3., (2019)

Tito Max Banegas Peña, Jorge Edison Becerra Molina; Edgar Geovanny Zamora Zamora; Héctor Espinoza Pillaga

un número de "restricciones del sistema" que la economía modelada debe satisfacer. Éstas incluyen tanto las condiciones de vaciado de los mercados como la selección del "cierre" macroeconómico para el modelo (Guedez, 2018).

\section{Ecuaciones de precio}

Por el lado de las importaciones, el modelo supone "país pequeño", por lo que los precios mundiales $\left(p w^{m}\right)$ son exógenos, Por el lado de las exportaciones, se admite una curva de demanda mundial de pendiente negativa y el precio mundial $\left(P W^{e}\right)$. En las ecuaciones (1) y (2), el precio doméstico de las importaciones $\left(P^{m}\right)$ y de las exportaciones $\left(P^{e}\right)$ es el precio mundial, que incluye el arancel o el subsidio a las exportaciones, multiplicado por el tipo de cambio $(R)$.

Las ecuaciones (3) y (4) describen los precios de las mercancías compuestas $Q$ y $X$ ( $P^{q}$ y $P^{x}$, respectivamente). $Q$ representa el agregado $C E S$ de las importaciones sectoriales $(M)$ y de los bienes domésticos ofrecidos al mercado doméstico $(D)$. $X$ representa la producción sectorial total, que es un agregado $C E T$ de bienes ofrecidos al mercado de exportación $(E)$ y bienes vendidos en el mercado doméstico $(D)$.

La ecuación (5) define el precio sectorial del valor agregado, o precio "neto" $\left(P^{v}\right)$, que es el precio del producto menos los impuestos indirectos unitarios $\left(t^{7}\right)$ y el costo unitario de los insumos intermedios (basado en los coeficientes fijos de insumo-producto, $a_{i j}$ ) El producto $P^{V} \cdot X$ es igual al valor agregado sectorial a precio de factor (Nuñez, 2012).

\section{Cuadro 2}

Ecuaciones de Precios

$P_{i}^{m}=p w_{i}^{m}\left(1+t_{i}^{m}\right) R$
$P_{i}^{e}=P W_{i}^{e}\left(1+t_{i}^{e}\right) R$
$P_{i}^{q}=\frac{P_{i}^{d_{*}} D+P_{i}^{m_{*} * M}}{Q}$
$P_{i}^{x}=\frac{P_{i}^{d^{d}}+P_{i}^{e} * E}{Q}$
$P_{i}^{x}=P_{i}^{x}\left(1-t_{i}^{x}\right)-\sum_{j} P_{j}^{q} * a_{j i}$
$P_{i}^{k}=\sum_{j} P_{j}^{q} * b_{j i}$
$P R I N D E X=\frac{G D P V A}{R G D P}$

Fuente: Tello Mario (2017)

Elaborado: Autores 


\section{Los modelos de equilibrio general dinámico estocásticos fundamentación microeconómica}

Vol. 3, núm. 3., (2019)

Tito Max Banegas Peña, Jorge Edison Becerra Molina; Edgar Geovanny Zamora Zamora;

Héctor Espinoza Pillaga

La ecuación (6) da el precio $\left(P^{k}\right)$ de una unidad de capital colocada en el sector $i$. Dado que la suma de cada columna de esta matriz es igual a uno, $P^{k}$ para cada sector es simplemente el promedio ponderado del costo unitario de los bienes de capital requeridos para crear una unidad de capital en cada sector de inversión.

Por último, la ecuación (7) define un índice de precios agregados (PINDEX), el cual se define a su vez como el deflactor del producto interno bruto o PIB (PIB nominal o GDPVA, dividido entre el PIB real o $R G D P$ ). Este índice proporciona el nivel de precios numerario contra el que se medirán los precios relativos del modelo.

\section{Ecuaciones de cantidad}

Las formas funcionales seleccionadas deben satisfacer ciertas restricciones de la teoría del equilibrio general. Las ecuaciones (8), (9) y (10) definen la tecnología de producción y la demanda de los factores. La ecuación (11) contiene las funciones de transformación CET, que combinan exportaciones y ventas domésticas, y la ecuación (12) muestra las funciones correspondientes de oferta de exportaciones, las cuales dependen de los precios relativos $\left(P^{e} / P^{d}\right)$. La ecuación (13) nos da la función de demanda de exportaciones mundiales para sectores en los que se supone que la economía tiene algún poder de mercado (y por lo tanto su curva de demanda adopta una pendiente negativa). Las ecuaciones (14) y (15) nos dan las funciones de agregación $C E S$, que describen cómo son demandados los productos domésticos y las importaciones, así como las funciones correspondientes de demanda de importaciones, las cuales dependen de los precios relativos $\left(P^{d} / P^{m}\right)$ mundiales para sectores en los que se supone que la economía tiene algún poder de mercado (y por lo tanto su curva de demanda adopta una pendiente negativa) (Nuñez, 2012).

\section{Cuadro 3}

Ecuaciones de Cantidad

$$
\begin{aligned}
& X_{i}=a_{i}^{D} \prod_{f} F D S C_{i f}^{\alpha t f}\left(F D S C_{i l}=\text { acervo de capital }\right) \\
& W F_{f} * w f d i s t_{i f}=P_{i}^{v} * \alpha_{i f} \frac{X_{i}}{F D S C_{i f}} \\
& I N T_{i}=\sum_{j} a_{i j} * X_{j} \\
& X_{i}=a_{i}^{T}\left[\gamma_{i} E_{i}^{p_{i}^{T}}+\left(1-\gamma_{i}\right) D_{i}^{p_{i}^{T}}\right]^{\frac{1}{p_{i}^{T}-1}} \\
& E_{i}=D_{i}\left[\frac{p_{i}^{e}\left(1-\gamma_{i}\right)}{P_{i}^{d} * \gamma_{i}}\right]^{\frac{1}{p_{i}^{T}}} \\
& E_{i}=e c o n_{i}\left[\frac{P W_{i}^{e}}{p w s e_{i}}\right]^{-\eta_{i}} \\
& Q_{i}=a_{i}^{c}\left[\delta_{i} M_{i}^{-p_{i}^{c}}+\left(1-\delta_{i}\right) D_{i}^{-p_{i}^{c}}\right]^{\frac{-1}{p_{i}^{c}}}
\end{aligned}
$$




\section{Los modelos de equilibrio general dinámico estocásticos fundamentación microeconómica}

Vol. 3, núm. 3., (2019)

Tito Max Banegas Peña, Jorge Edison Becerra Molina; Edgar Geovanny Zamora Zamora; Héctor Espinoza Pillaga

$$
M_{i}=D_{i}\left[\frac{P_{i}^{d} * \delta_{i}}{P_{i}^{m}\left(1-\delta_{i}\right)}\right]^{-\frac{1}{1+p_{i}^{c}}}
$$

Fuente: Tello Mario (2017)

Elaborado: Autores

Para estar completa, la función de producción [ecuación (8)] debe incluir todos los insumos como argumentos: capital, trabajo e insumos intermedios. Las condiciones de demanda para factores en la ecuación (9) se escribirían entonces de la siguiente manera (dejando a un lado los subíndices sectoriales):

$$
\begin{gathered}
\text { Precio para factores }=\text { Ingreso marginal del producto } \\
=\left(1-t^{X}\right) * p x * \frac{\partial X}{\partial F}
\end{gathered}
$$

Donde $F$ es el conjunto completo de insumos para factores. El anidamiento descrito anteriormente se tomaría en cuenta al usar la regla de la cadena. En la ecuación (8) especificamos, en cambio, la función de producción sólo como una función de los factores primarios, definidos como capital y trabajo. Las demandas de insumos intermedios se dan en la ecuación (10), mientras que la ecuación (9) muestra la demanda de factores primarios en la siguiente forma (de nuevo, dejando a un lado los subíndices sectoriales):

$$
\text { Precios de los factores }=P^{v} * \frac{\partial X}{\partial F D S C}
$$

Donde $F D S C$ se refiere ahora únicamente a los factores primarios, y $P^{v}$ es el precio de valor agregado [ecuación (5)], el cual se define neto a partir de los impuestos indirectos y de los costos de insumos intermedios. Este tratamiento es equivalente a escribir el conjunto completo de funciones anidadas y sus derivadas correspondientes. El enfoque utilizado aquí es más simple y se ha vuelto tradicional en muchos modelos MEGA.

\section{Ecuaciones de ingreso}

El cuadro 3 presenta las ecuaciones que mapean el flujo de ingreso de valor agregado hacia las instituciones y, en última instancia, hacia los hogares. La ecuación (16) define los ingresos de los factores, los cuales a su vez son distribuidos entre los hogares de capitalistas y de trabajadores en las ecuaciones (17) y (18).

Las ecuaciones (19), (20) y (21) determinan los ingresos del gobierno por aranceles (TARIFF), por impuestos indirectos (INDTAX) y por impuestos sobre la renta (HHTAX). La ecuación (22) resume los subsidios a la exportación por sector (EXPSUB). 


\section{Los modelos de equilibrio general dinámico estocásticos fundamentación \\ microeconómica}

Vol. 3, núm. 3., (2019)

Tito Max Banegas Peña, Jorge Edison Becerra Molina; Edgar Geovanny Zamora Zamora;

Héctor Espinoza Pillaga

\section{Cuadro 4}

Ecuaciones de ingreso

$$
\begin{aligned}
& Y_{f}^{F}=\sum_{i} W F_{f} * \text { FDSC }_{i f} * \text { wfdist }_{i f} \\
& Y_{\text {cap } H}^{H}=Y_{1}^{F}-D E P R E C \quad\left(Y_{1}^{F}=\text { ingreso del factor capital }\right) \\
& Y_{\text {trabeh }}^{H}=\sum_{f \neq j} Y_{f}^{F} \\
& \text { TARIFF }=\sum_{i} p w_{i}^{m} * M_{i} * t_{i}^{m} * R \\
& \text { INDTAX }=\sum_{i} P_{i}^{x} * X_{i} * t_{i}^{x} \\
& \text { HHTAX }=\sum_{h} Y_{h}^{H} * t_{h}^{h} \quad(h=\text { capital, trabajo }) \\
& \text { EXPSUB }=\sum_{i} P W_{i}^{e} * E_{i} * t_{i}^{e} * R \\
& \text { GR=TARIFF+INDTAX+HHTAX-EXPSUB } \\
& \text { DEPREC }=\sum_{i} d e p r^{i} * P_{i}^{k} * F D S C_{i 1} \quad\left(F D S C_{i 1}=\text { acervo de capital }\right) \\
& \text { HHSAV }=\sum_{h} Y_{h}^{H} *\left(1-t_{h}^{H}\right) * m_{h} \\
& \text { GOVSAV }=G R-\sum_{i} P_{i}^{q} * G D_{i} \\
& \text { SAVING }=H H S A V+G O V S A V+D E P R E C+F S A V * R
\end{aligned}
$$

Fuente: Tello Mario (2017)

Elaborado: Autores

Mientras que los ingresos gubernamentales (GR) se obtienen como la suma de éstos en la ecuación (23). Los componentes del ahorro incluyen la depreciación financiera (DEPREC) en la ecuación (24), el ahorro de los hogares (HHSAV) con propensiones al ahorro fijas $\left(\mathrm{mpsh}_{\mathrm{k}}\right)$ en la ecuación (25) y el ahorro del gobierno (GOVSAV) en la ecuación (26), obtenido como la diferencia entre el ingreso del gobierno y su consumo. El ahorro total (SAVING) en la ecuación (27) incluye estos tres elementos domésticos más el ahorro extranjero en moneda doméstica $(\mathrm{FSAV} * \mathrm{R})$. 


\section{Los modelos de equilibrio general dinámico estocásticos fundamentación microeconómica}

Vol. 3, núm. 3., (2019)

Tito Max Banegas Peña, Jorge Edison Becerra Molina; Edgar Geovanny Zamora Zamora; Héctor Espinoza Pillaga

Estas ecuaciones de ingresos incluyen tres balances macroeconómicos: el balance ahorro-inversión, el déficit gubernamental y la cuenta corriente. Las empresas y los hogares ahorran proporciones fijas (depr y mps) de sus ingresos, el ahorro del gobierno lo constituyen el superávit o el déficit presupuestal y el ahorro externo representa la entrada de capital necesaria para equilibrar los pagos internacionales, esto es, el ahorro extranjero neto. Dado que el modelo satisface la ley de Walras, los tres balances macroeconómicos deben satisfacer la identidad:

$$
\text { Ahorro privado + ahorro del gobierno + ahorro extranjero = inversión }
$$

\section{Ecuaciones de gasto}

El cuadro 4 proporciona las ecuaciones que completan el flujo circular en la economía, determinando la demanda de bienes por parte de los diversos actores. El consumo privado (CD) se obtiene en la ecuación (28) sumando las demandas de los hogares determinadas mediante el uso de participaciones de gasto fijas (bs) y netas de los impuestos a su ingreso (t). En la ecuación (29), la demanda del gobierno (GD) por bienes finales se define utilizando participaciones fijas del gasto real agregado en bienes y servicios (gdtot). La demanda y los cambios de inventarios (DST) se determinan en la ecuación (30) utilizando participaciones fijas de la producción sectorial (dstr). La inversión nominal fija agregada (FXDINV) se calcula en la ecuación (31) como la inversión total (INVEST) menos la acumulación de inventarios. La inversión fija agregada se convierte en inversión sectorial real por sector de destino (DK) en la ecuación (32) utilizando participaciones nominales fijas (kshr), las cuales suman 1 cuando se consideran todos los sectores (Nuñez, 2012).

\section{Cuadro 5}

Ecuaciones de gasto

$$
\begin{aligned}
& P_{f}^{F} C D_{i}=\sum_{h}\left[\beta_{i h}^{H} * Y_{h}^{H} *\left(1-m p s_{h}\right) *\left(1-t_{h}^{H}\right)\right] \\
& G D_{i}=\beta_{i}^{G} * g d t o t \\
& D S T_{i}=d s t r_{i} * X_{i} \\
& F X D I N V=I N V E S T-\sum_{i} p_{i}^{q} * D S T_{i} \\
& P_{i}^{k} * D K_{i}=k s h r_{i} * F X D I N V \\
& I D_{i}=\sum_{j} b_{i j} * D K_{j} \\
& G D P V A=\sum_{i} P_{i}^{v} * X_{i}+I N D T A X+T A R I F F-E X P S U B \\
& R G D R=\sum_{i}\left(C D_{i}+G D_{i}+I D_{i}+D S T_{i}+E_{i}-p w_{i}^{m} * M_{i} * R\right)
\end{aligned}
$$

Fuente: Tello Mario (2017)

Elaborado: Autores 


\section{Los modelos de equilibrio general dinámico estocásticos fundamentación microeconómica}

Vol. 3, núm. 3., (2019)

Tito Max Banegas Peña, Jorge Edison Becerra Molina; Edgar Geovanny Zamora Zamora;

Héctor Espinoza Pillaga

La ecuación (33) transforma la inversión por sector de destino en demanda de bienes de capital por sector de origen (ID), utilizando la matriz de composición de capital $\left(\mathrm{b}_{\mathrm{ij}}\right)$.

Las ecuaciones (34) y (35) definen los PIB nominal y real, los cuales se utilizan para calcular el deflactor del PIB especificado como numerario en la ecuación de precio (7). El PIB real (RGDP) se define a partir del lado del gasto, con las importaciones valuadas en precios mundiales (el precio mundial multiplicado por el tipo de cambio). El PIB nominal (GDPVA) se genera del lado del valor agregado (Nuñez, 2012).

\section{Condiciones para el vaciado de los mercados}

El cuadro 5 contiene ecuaciones que definen las restricciones de sistema que el modelo MEGA debe satisfacer. A pesar de que se reconoce que el modelo es de equilibrio general, con todas las variables endógenas determinadas de manera conjunta, es sin embargo útil pensar en relacionar cada una de estas condiciones de equilibrio con una "variable equilibrante". En una economía de mercado competitiva, estas condiciones de equilibrio corresponden a condiciones para que los mercados se "vacíen", con precios que se ajustan para vaciar cada mercado (Nuñez, 2012).

La ecuación (36) especifica que la oferta sectorial de mercancías compuestas $(Q)$ debe ser igual a la suma de cada uno de los componentes de la demanda, y así define el equilibrio o "vaciado" en los mercados de productos. También hay una ecuación sectorial análoga para el vaciado de los mercados para bienes producidos domésticamente y vendidos en el mercado doméstico $(D)$. Sin embargo, partiendo de la ecuación (15) resulta evidente que la proporción de importaciones y ventas domésticas es la misma para todas las categorías de importaciones. De esta manera, en el nivel sectorial el hecho de especificar una condición separada de vaciado de los mercados para bienes producidos domésticamente y vendidos en el mercado doméstico equivale a multiplicar ambos lados de la ecuación (36) por la razón $D_{i} / Q_{i}$. Dado que, si la ecuación (36) se mantiene, también lo hará esta nueva ecuación en la que ambos lados se multiplican por el mismo número; no se requiere una ecuación separada.

\section{Cuadro 6}

Condiciones de equilibrio o de vaciado de los mercados y cierre macroeconómico

$$
\begin{aligned}
& Q_{i}=I N T_{i}+C D_{i}+G D_{i}+I D_{i}+D S T_{i} \\
& \sum_{i} F D S C_{i f}=f s_{f} \\
& p w_{i}^{m} * M_{i}=P W_{i}^{e} * E_{i}+F S A V \\
& S A V I N G=I N V E S T
\end{aligned}
$$

Fuente: Tello Mario (2017)

Elaborado: Autores 


\section{Los modelos de equilibrio general dinámico estocásticos fundamentación microeconómica}

Vol. 3, núm. 3., (2019)

Tito Max Banegas Peña, Jorge Edison Becerra Molina; Edgar Geovanny Zamora Zamora; Héctor Espinoza Pillaga

Las variables equilibrantes para la ecuación (36) son los precios sectoriales. Hay nueve precios en el modelo (cada uno de los cuales tiene subíndices sectoriales): $\mathrm{pw}^{\mathrm{m}}, \mathrm{PW}^{\mathrm{e}}, \mathrm{P}^{\mathrm{m}}, \mathrm{P}^{\mathrm{e}}, \mathrm{P}^{\mathrm{q}}, \mathrm{P}^{\mathrm{x}}, \mathrm{P}^{\mathrm{v}}, \mathrm{Pd}$ y $\mathrm{P}^{\mathrm{k}}$. Los precios mundiales (pwrll y PWe) se tratan separadamente. De los siete precios restantes, seis aparecen en el lado izquierdo de las ecuaciones de precio (de la 1 a la 6), dejando a $\mathrm{P}^{\mathrm{d}}$ como la variable "libre" para ajustar.

La ecuación (37) define el equilibrio en los mercados de factores. Las ofertas de factores primarios lfs J\} se fijan de manera exógena. El vaciado de mercados requiere que la demanda total de los factores sea igual a la oferta, y las variables equilibrantes son los precios promedio de los factores $\left(\mathrm{fs}_{\mathrm{f}}\right)$. En el modelo especificado aquí, todos los factores primarios son intersectorialmente móviles: las demandas de los factores se determinan mediante la ecuación (9), el vaciado de los mercados se logra cambiando los precios de los factores (WFf) junto con los parámetros sectoriales específicos exógenos (wfdistif).

Las dos ecuaciones restantes describen las condiciones de equilibrio macroeconómico para la balanza de pagos y el balance ahorro-inversión. En la ecuación (38) la balanza de pagos: el ahorro externo (FSAV) es la diferencia entre las importaciones y las exportaciones totales.

La condición final de cierre macroeconómico en la ecuación (39) requiere que el ahorro agregado sea igual a la inversión agregada. Los componentes del ahorro total: el ahorro del gobierno se determina como el residuo después de que el ingreso del mismo es gastado en un consumo fijo real (gdtot), el ahorro privado se determina por las tasas fijas de ahorro, y el ahorro extranjero (por lo menos en una opción de cierre) se fija de manera exógena (Nuñez, 2012).

\section{Conclusiones.}

Los modelos de equilibrio General Computable son sistemas de ecuaciones que utilizan la economía que una unidad funcional que las relaciones entre los diferentes agentes (Burfisher, 2011), es decir, estos modelos como cada agente en la economía representa un sistema de ecuaciones interdependientes, lo que hace referencia a que cada uno de los agentes que se han visto afectados por las decisiones de consumo y producción de otro. Cicowiez y Di Gresia (2004) definen la lógica de un MEGC como una representación computable de la economía compuesta por diversos agentes, quienes actúan de acuerdo con los principios de la racionalidad microeconómica. Por lo general, los agentes que interactúan en los diferentes mercados son: las familias, las empresas, el gobierno y el sector externo (Heckscher \& Ohlin, 1990).

Para el desarrollo de un MEGC es de vital importancia contar con información sobre las relaciones entre los agentes, por qué es primordial Contar una Matriz de Contabilidad Social (MCS), la cual es el insumo más importante para el desarrollo de estos modelos debido a La cantidad de información que contiene.

Los MEGC deben tener los siguientes elementos (Burfisher 2011):

$\checkmark$ Conjuntos: Los conjuntos hacen referencia al dominio sobre el cual se cimienta la construcción del modelo. Por lo general, los conjuntos hacen referencia a los consumidores $i$, las firmas $j$ y los bienes $l$. Por ejemplo, un modelo con dos consumidores, dos firmas y dos bienes, se denota como un modelo $2 \times 2 \times 2$.

$\checkmark$ Variables endógenas: Una variable endógena es aquella que determina la solución de un sistema. En los MEGC, estas variables están representadas por los precios y las cantidades de equilibrio. 


\section{Los modelos de equilibrio general dinámico estocásticos fundamentación microeconómica}

Vol. 3, núm. 3., (2019) Tito Max Banegas Peña, Jorge Edison Becerra Molina; Edgar Geovanny Zamora Zamora; Héctor Espinoza Pillaga

$\checkmark \quad$ Variables exógenas: Las variables exógenas son aquellas que tienen valores fijos y, por ende, no cambian con la solución del modelo.

$\checkmark$ Cierre macro: Un cierre hace referencia a que el investigador debe decidir cuáles variables son endógenas y cuáles son exógenas. Algunos de los ejemplos más comunes en este aspecto son la decisión a la que se enfrenta el investigador a la hora de determinar qué tipo de variable representa la oferta de trabajo en un momento determinado y la libertad de elegir qué variable entre el ahorro o la inversión (o ambas) debe ser exógena con el objetivo de establecer la igualdad en esta identidad macroeconómica.

$\checkmark$ Parámetros: Tal como las variables exógenas, los parámetros también representan valores que no varían con el paso del tiempo. Burfisher (2011) afirma que sólo existen tres tipos de parámetros, los cuales son: las tarifas impositivas, las elasticidades de oferta y demanda (Por ejemplo, los $\alpha$ en la función de utilidad) y los parámetros de participación (por ejemplo, el valor de la tecnología $A$ en la función de producción).

$\checkmark$ Calibración: El proceso de calibración se define como el método utilizado para calcular los valores de los parámetros, el cual consiste en solucionar un conjunto de ecuaciones fijando el valor de las variables endógenas por aquel registrado en la MCS, dicha situación se define como el año base, el cual debe estar en equilibrio, con el fin de despejar el valor de los parámetros.

$\checkmark$ Ecuaciones: Las ecuaciones son uno de los elementos más importantes para la construcción de un MEGC, debido a que a través de estas se representa el comportamiento de los agentes en el mercado. Por ejemplo, el problema de las firmas consiste en minimizar sus costos o maximizar sus beneficios, mientras que el problema el consumidor consiste en maximizar su utilidad o minimizar su gasto. La elección de las funciones cuyo objetivo es representar un comportamiento debe realizarse en función a las preferencias de la unidad de análisis, es decir, la forma funcional debe estar de acuerdo al comportamiento de los datos observados. Además, con el fin de representar el flujo de la economía, todas las ecuaciones deben relacionarse (directa o indirectamente) para representar la oferta y la demanda. Por ejemplo, al remitirnos a la definición de equilibrio, suponemos que la oferta debe ser igual a la demanda en todos los mercados, por lo cual las ecuaciones deben representar dicho equilibrio.

$\checkmark$ Numerario: es importante tener en cuenta que los MEGC trabajan con precios relativos y, precisamente, el numerario hace referencia a aquel precio de un bien que es tomado como 1, con el objetivo de que la comparación relativa sea realizada en torno de él, es decir, el numerario básicamente es una unidad de medida. Por ejemplo, si los salarios son tomados como el numerario, unos aumentos en los precios de los bienes de consumo se deben analizar de forma relativa con respecto a los salarios.

Para la elaboración de un MEGC, Cicowiez y Di Gresia (2004) describen 7 secciones o pasos suficientes para la construcción del mismo, los cuales son:

(1) Se deben determinar las dimensiones básicas del modelo, es decir, es necesario establecer el número de agentes $i$, el número de firmas $j$ y la cantidad de bienes en la economía $l$. Así, el modelo presenta una dimensión $i * j * l$. 


\section{Los modelos de equilibrio general dinámico estocásticos fundamentación microeconómica}

Vol. 3, núm. 3., (2019)

Tito Max Banegas Peña, Jorge Edison Becerra Molina; Edgar Geovanny Zamora Zamora; Héctor Espinoza Pillaga

(2) Luego de haber determinado las dimensiones básicas del modelo, es necesario definir el problema de elección al cual se van a enfrentar cada uno de los agentes. Específicamente, esta sección trata de establecer las formas funcionales para el problema del productor y las preferencias para el problema del consumidor.

(3) Se debe contar con una MCS consistente y balanceada con el fin de representar el punto de partida del modelo.

(4) Una vez se han establecido las formas funcionales, es necesario obtener los valores de los parámetros y de las variables exógenas, con el objetivo de simular el estado base de la economía.

(5) Los MEGC están compuestos por grandes sistemas de ecuaciones los cuales deben solucionarse a través de métodos numéricos. Por esta razón, se debe codificar el sistema de ecuaciones en un software de programación matemática con el fin de encontrar una solución factible para los experimentos de política pública. Por lo general, GAMS (General Algebraic Modeling System) es el software recomendado para realizar este tipo de codificaciones.

(6) Se debe replicar el caso base utilizando el sistema de ecuaciones especificado y empleando los parámetros y variables exógenas.

Por último, una vez cumplidos los pasos anteriores, es posible realizar simulaciones modificando el equilibrio inicial, con el objetivo de analizar el comportamiento de los agentes y sus interacciones hasta encontrar un nuevo equilibrio en la economía. Las simulaciones se definen como experimentos controlados.

\section{Bibliografía.}

Criollo, D. (2010). Un Modelo de Equilibrio General Dinámico para el Análisis de Políticas Económicas de la Economía Ecuatoriana. Escuela Politécnica Nacional, Escuela de Ciencias, Quito - Ecuador.

Chiang, A. (2006). Métodos Fundamentales de Economía Matemática. McGrawHill Iberoamericana, México D.F. ISBN - 13: 978-970-10-5614-1.

Guedez, G. (2018). Efecto Macroeconómico y Sectorial del Gasto Público en Venezuela: Un estudio a través de un Modelo de Equilibrio General Dinámico Estocástico. Universidad Católica Andrés Bello, Dirección General de Postgrados, Caracas - Venezuela.

Haro, R. (2008). Metodologías para la estimación matemática de la matriz de insumo-producto simétrica. Instituto Nacional de Estadística Geografía e Informática INEGI, México D.F., ISBN: 978-968-5696-25-8.

Krugman, P. (2014). Macroeconomía. Editorial Reverte S.A., Basauri - España. ISBN: 978-84-291-2604-4.

Lozano, C. (2004). Elasticidades de sustitución Armington para Colombia. Archivos 


\section{Los modelos de equilibrio general dinámico estocásticos fundamentación microeconómica}

Vol. 3, núm. 3., (2019)

Tito Max Banegas Peña, Jorge Edison Becerra Molina; Edgar Geovanny Zamora Zamora; Héctor Espinoza Pillaga

de Economía, Dto. 271. Pág. 2-15. Recuperado el 15 de enero de 2018 de https: //www.researchgate.net /publication/5007298 Elasticidades_de_sustitucion_Armington_para_Colombia.

Nicholson, W. y Snyder, Ch. (2011). Microeconomía Intermedia y su Aplicación.

CENGAGE Learning's Editores, México D.F. ISBN: 978-0-324-59910-7.

Nuñez, G. (2012). Equilibrio General Aplicado: Metodología e Investigación. Editorial académica española, Saarbrucken - Alemania, ISBN: 978-3-8473-6472-6.

Pedauga, L., Sáez, F. y Velázquez, A. (2012). Técnicas para la Simulación de Equilibrio General Computable mediante el uso de GAMS: Aplicaciones al caso venezolano. BCV Banco Central de Venezuela, Serie Documentos de Trabajo No 132. Caracas - Venezuela.

Pérez, W. y Acota, M. Lozano, C. (2005). Modelo Ecuatoriano de Equilibrio General Aplicado (MEEGA). Cuestiones Económicas Vol. 22, No 2:2-3, 2005. Recuperado el 12 de febrero de 2018 de https://www.bce.fin.ec/cuestiones_economicas/images/PDFS/2005/No2/Vol.22-22005WilsonPerez.pdf.

SanchPez, L. Ramirez, J. (2005). Modelo de Equilibrio General de Ecuador: Extensión en el Análisis del Impacto Regional. Cuestiones Económicas Vol. 22, No 2:2-3, 2005. Recuperado el 12 de febrero de 2018 de https: // www.bce.fin.ec/cuestiones economicas /images /PDFS /2005 /No2 /Vol.22-2-2005Sanchez y Ramírez Analisisdeimpactoregional.pdf.

Tello, M. (2017). Análisis de Equilibrio General: Modelos y aplicaciones para países en desarrollo. Pontificia Universidad Católica del Perú, Fondo Editorial, Lima - Perú, ISBN: 978612-317-224-4. 